\title{
Influencia de la separación de agua residual porcina en fracciones sólida y líquida, en la producción de metano con lodo anaerobio granular y disperso
}

\author{
Influence of swine wastewater separation into solid and liquid fractions, on methane production with \\ granular and disperse sludge
}

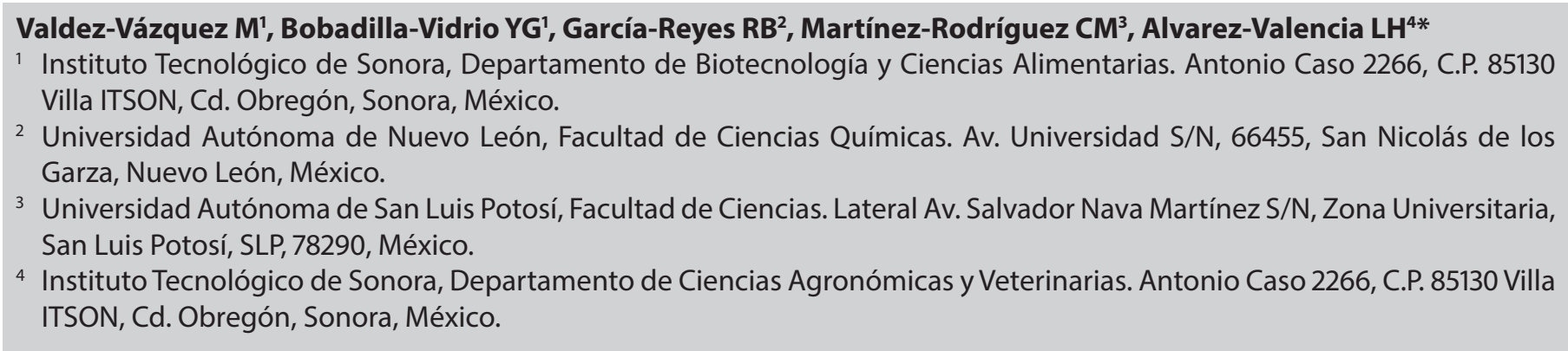

\section{RESUMEN}

Las estrategias actuales de tratamiento de efluentes porcinos en México son en muchos casos poco eficientes, que se traduce en la descarga de aguas tratadas parcialmente y no tratadas, así como el desaprovechamiento de un recurso energético. El objetivo de este estudio fue evaluar el impacto de la separación sólida-líquida del agua residual porcina sobre la producción de metano, utilizando lodo granular y lodo disperso, bajo tres concentraciones. La concentración de DQO en el efluente crudo (EC) fue de 13,640 mg/L, con 69 $\%$ correspondiente a la fracción sólida (FS) y el resto para la fracción líquida (FL). Los resultados indican que los cultivos con la FS produjeron una mayor cantidad de metano, incrementándose 1.47, 1.31 y 1.22 veces en comparación al EC, para las concentraciones de 3, 6 y $9 \mathrm{~g} \mathrm{SSV/L}$ de lodo disperso. La estrategia de separación sólida-líquida resultó en incrementos en la producción de metano, evidenciado porque la suma del metano acumulado por la FS y FL fue entre 2.14 y 2.28 veces mayor que la cantidad obtenida por el EC. La aplicación de procesos anaerobios para tratar residuos sólidos y líquidos permitirá recuperar de manera más eficiente la energía a partir de la transformación de la materia orgánica a metano.

Palabras clave: agua residual porcina, metano, separación de sólidos, lodo granular, lodo disperso

\section{ABSTRACT}

In most cases, the current strategies to treat swine effluents in Mexico are not efficient, resulting in the discharge of partially treated wastewater, wasting an energy resource. This study aimed to evaluate the impact of the solid-liquid separation of swine wastewater on methane production using granular (GS) and disperse sludge (DS), under three concentrations. The COD concentration in the raw effluent (RE) was 13,640 $\mathrm{mg} / \mathrm{L}$, which corresponds up to

*Autor para correspondencia: Luis H. Alvares Valencia

Correo electrónico: luis.alvarez@itson.edu.mx

Recibido: 15 de julio de 2021

Aceptado: 7 de enero de 2022
$69 \%$ to the solid fraction (SF), and the remaining to the liquid fraction (LF). The results indicate that the cultures with the SF produced the higher cumulative methane, increasing 1.47-, 1.31 -, and 1.22-times in retaliation to the $\mathrm{RE}$, at 3, 6, and $9 \mathrm{~g}$ VSS/L of DS. The solid-liquid separation strategy resulted in a higher methane production, evidenced by the amount of methane obtained by the SF and LF, which was, respectively, 2.14- and 2.28-times higher than the obtained by the RE. The application of anaerobic processes to treat solid and liquid wastes will allow to recovery efficiently the energy from the transformation of organic matter into methane.

Key words: swine wastewater, methane, solid separation, granular sludge, disperse sludge

\section{INTRODUCCIÓN}

México se ubica en la novena posición a nivel mundial en producción de carne de cerdo con una participación de 1.4 \% en el año 2019, alcanzando 1.6 millones de toneladas de carne en canal y crecimiento anual de $4.9 \%$. La producción en el año 2019 se concentra en seis entidades del país que en conjunto producen $77.1 \%$ : Jalisco (21.4\%), Sonora (19.3\%), Puebla (10.7\%), Yucatán (9.2\%), Veracruz (8.9\%) y Guanajuato (7.7 \%). Los principales municipios a nivel nacional son Navojoa, Hermosillo y Cajeme, los tres ubicados en Sonora, con una producción cercana a 10,000 millones de pesos (FIRA, 2020).

De manera paralela al beneficio económico, la producción intensiva de carne de cerdo se ha asociado a diferentes problemas ambientales que comprometen la calidad del agua, suelo y aire, tales como eutrofización, acidificación, cambio de uso de suelo y cambio climático (Dourmad et al., 2014). El principal desecho generado por esta actividad son las aguas residuales, que se caracterizan por contener altas concentraciones de materia orgánica, sólidos totales, nitrógeno y fósforo (Boursier et al., 2005)y hormonas (Hanselman et al., 2003). La concentración de materia orgánica en 
las aguas residuales varía según el tamaño de la granja, alcanzando entre 3,500 y 9,300 mg DQO/L en granjas pequeñas (hasta 2,500 cerdos) y entre 34,000 y $40,500 \mathrm{mg} \mathrm{DQO} / \mathrm{L}$ en granjas grandes ( $\geq 8,000$ cerdos) (Garzón-Zúñiga y Buelna, 2014). La cantidad de materia orgánica en este tipo de efluentes representa un potencial grande de ser utilizado como fuente de energía alterna mediante la obtención de biogás utilizando digestión anaerobia. En una estimación hecha considerando la capacidad instalada de crianza de cerdos en el estado de Sonora, arrojó que la cantidad de residuos que potencialmente se pueden generar permitiría la generación de $127,638 \mathrm{~m}^{3} \mathrm{CH}_{4}$ /día, que equivale a 525,216 kWh (Alvarez et al., 2019).

A pesar del potencial, los esfuerzos para recuperar la energía a partir de los residuos porcinos son escasos o nulos, lo cual es evidenciado por la carencia o ineficiencia de los sistemas de tratamientos en México. En la mayoría de los casos, los sistemas que se han instalado en las granjas son: 1) fosa de homogenización, 2) digestor anaerobio de una mezcla de líquidos y sólidos, 3) lagunas facultativas o de otro tipo, o bien una combinación de estos procesos (Garzón-Zúñiga y Buelna, 2014). Además, en la mayoría de los sistemas donde hay producción de biogás, en los casos que se utiliza un proceso anaerobio, no se aprovecha para la generación de calor o electricidad. Para mejorar el proceso de digestión anaerobia y en consecuencia la recuperación de energía a partir de los residuos porcinos en México, es necesario implementar sistemas de tratamiento como los reactores anaerobios de flujo ascendente (tipo UASB) o reactores completamente mezclados (tipo CSTR). Sin embargo, el proceso de digestión anaerobia también presenta algunas limitaciones debido a la naturaleza del sustrato y en general de los componentes del agua residual porcina. Por ejemplo, la hidrólisis, paso inicial de la digestión anaerobia, es comúnmente afectada por la presencia de sólidos orgánicos complejos, en tanto la metanogénesis puede limitarse por la disponibilidad de sustratos orgánicos solubles (Tomei et al., 2009). En estudios previos se ha observado que la estrategia de separar los sólidos de los efluentes y su posterior tratamiento mediante procesos anaerobios, ha mejorado la digestión de la fracción sólida (FS) (Cestonaro do Amaral et al., 2016) y la fracción líquida (FL) (Yang et al., 2015). Este comportamiento puede estar influenciado por la presencia de compuestos inhibitorios en los residuos. Sin embargo, en esos estudios no establecen una comparación con el tipo y concentración de inóculo utilizado. El objetivo de este estudio fue evaluar el impacto de la separación sólida-líquida del agua residual de una granja porcina sobre la producción de metano, utilizando cultivos en lote inoculados con distintas concentraciones de lodo granular y lodo disperso, para su aplicación en reactores UASB y CSTR, respectivamente.

\section{MATERIALES Y MÉTODOS}

\section{Agua residual porcina e inóculo anaerobio}

La muestra de agua residual se colectó en una granja porcina ubicada en el municipio de Bácum, Sonora. Una por- ción del efluente crudo (EC) fue centrifugada a 4,000 rpm durante $15 \mathrm{~min}$. El sobrenadante representa la $\mathrm{FL}$ y el sedimento colectado fue resuspendido en la porción de agua destilada que representa a la FL para mantener la concentración inicial, y esta fracción representa a la FS. Las tres muestras fueron analizadas para determinar la DQO y $\mathrm{pH}$, utilizando métodos reportados en standard methods (APHA, 2005). Sé utilizó lodo granular metanogénico como inóculo que fue colectado de un reactor anaerobio de flujo ascendente (UASB), instalado en una industria cervecera localizada en Cd. Obregón Sonora. Una porción del lodo granular, con tamaño de gránulo de 2-4 mm, fue disgregado con un tamiz de 0.3 $\mathrm{mm}$ para obtener el lodo disperso. Ambos lodos contenían $9.5 \%$ de sólidos suspendidos volátiles (SSV) y no fueron previamente aclimatados en aguas residuales porcinas u otra condición en el laboratorio.

\section{Cultivos en lote para producción de metano}

Las cinéticas de producción de metano se realizaron por triplicado en botellas serológicas de $120 \mathrm{~mL}$ con un volumen de trabajo de $50 \mathrm{~mL}$ de cada una de las fracciones de agua residual. Las concentraciones de lodo granular disperso utilizadas como inóculo fueron 3,6 y $9 \mathrm{~g} \mathrm{SSV/L}$. Se utilizó un tapón de hule y arillo de aluminio para sellar los cultivos; después, se utilizó gas helio para desplazar el aire (oxígeno) del espacio de cabeza de cada botella, a fin de crear condiciones anaerobias y promover la metanogénesis. Posteriormente, las botellas se colocaron en una incubadora con agitación a $40^{\circ} \mathrm{C}$ y $150 \mathrm{rpm}$. Periódicamente, durante los 30 días que duró la incubación se midió el volumen de metano producido utilizando el método de desplazamiento de líquido, con una solución de $\mathrm{NaOH}$ al $2 \%$ que permite la solubilidad del $\mathrm{CO}_{2}$.

Los valores experimentales de producción metano fueron utilizados para determinar los parámetros cinéticos del proceso mediante el modelo modificado de Gompertz:

$$
M(t)=M_{\max } * \exp \left[-\exp \left[\frac{2.71828 * R_{\max }}{M_{\max }}(\lambda-t)+1\right]\right]
$$

donde $M(t)$ es el volumen $(\mathrm{mL})$ de metano en un tiempo determinado, $t$ es el tiempo (días), $M_{\max }$ es la cantidad máxima de metano producido $(\mathrm{mL}), R_{\max }$ es la tasa máxima de producción de metano ( $\mathrm{mL} /$ día) y $\lambda$ es la fase lag (días). Tanto los parámetros del modelo Gompertz, así como un análisis de varianza y prueba Tukey se realizaron con Minitab 19 para determinar diferencias entre las medias $(p<0.05)$.

\section{RESULTADOS Y DISCUSIÓN} Influencia de la separación sólida-líquida sobre la DQO y pH

El agua residual y sus fracciones fueron analizadas para determinar el contenido de materia orgánica expresado como DQO (Tabla 1). La concentración de materia orgánica en el EC fue de 13,640.0 mg DQO/L. De esta concentración, el $69 \%$ corresponde a la FS (9,413 mg DQO/L) y el porcentaje restante corresponde a la $\mathrm{FL}$ (3,685 mg DQO/L). La suma de la 
Tabla 1. Contenido de materia orgánica expresada como DQO y valores de $\mathrm{pH}$ para los diferentes tipos a agua residual.

Table 1. Organic matter content determined as $\mathrm{COD}$ and $\mathrm{pH}$ values for the three types of wastewaters.

\begin{tabular}{lcl}
\hline Tipo de agua & DQO $(\mathbf{m g} / \mathbf{L})$ & pH \\
\hline Efluente crudo & $13640.0 \pm 1501.7$ & 6.8 \\
\hline Fracción sólida & $9413.3 \pm 1759.7$ & 6.5 \\
\hline Fracción líquida & $3685.0 \pm 157.4$ & 6.6 \\
\hline
\end{tabular}

DQO determinada en las FC y FL corresponde en general a la $\mathrm{DQO}$ total en el EC. La concentración de DQO en este tipo de efluentes es muy variable, presentando valores desde 6,000 a 50,000 mg/L (Boursier et al., 2005), por lo que la muestra utilizada en este estudio contiene una DQO que se ubica en un rango bajo. Resulta interesante el hecho de que la eliminación física de los sólidos contenidos en el agua bajo estudio puede disminuir hasta el $69 \%$ de la DQO, lo que se traduce en un agua que puede ser más fácilmente tratada. Actualmente en México son inexistentes o casi nulas las estrategias de tratamiento primario en granjas de cerdo encaminadas a eliminar sólidos mediante procesos de separación física. Normalmente los efluentes se conducen hacia los tratamientos secundarios en donde se utilizan lagunas anaerobias o facultativas medianamente tecnificadas (Garzón-Zúñiga y Buelna, 2014). Por otro lado, la separación sólido-líquida no tuvo un efecto considerablemente importante sobre el $\mathrm{pH}$, ya que el valor en el EC fue 6.8 y bajó a 6.5 en la FS y a 6.6 en la FL. Esta ligera disminución en el pH pudiera no representar un efecto adverso sobre los microrganismos involucrados en la metanogénesis, puesto que el rango óptimo de $\mathrm{pH}$ para que este proceso ocurra es 6.8 a 7.2 , pero puede tolerar de 6.5 hasta 8.0 (Cioabla et al., 2012).

\section{Influencia de la separación sólida-líquida sobre la pro- ducción de metano}

La producción de metano a partir de la digestión anaerobia de la materia orgánica contenida en el agua residual porcina ocurrió bajo todas las condiciones probadas (Figura 1). La mayor cantidad de metano acumulado fue con la FS, seguida por el EC y la FL, ocurrida bajo las tres concentraciones de inóculo probadas. Por ejemplo, con el lodo disperso y utilizando la FS se obtuvo 1.47, 1.31 y 1.22 veces más metano acumulado que lo obtenido con el EC, para 9, 6 y 3 g SSV/L, respectivamente. Además, en los tres tipos de agua residual, los valores acumulados de metano resultaron más altos con el lodo disperso en comparación con el lodo granular. La concentración de inóculo impactó la producción de metano. Por ejemplo, el metano producido con la FS utilizando lodo disperso a $9 \mathrm{~g} \mathrm{SSV/L}$ fue $318.8 \mathrm{~mL}$, que es 1.12 - y 1.27 -veces más alto a lo obtenido con 6 y $3 \mathrm{~g} \mathrm{SSV/L}$, respectivamente, después de 26 h de incubación. Para el EC y la FL también hubo una mejor producción de metano con el lodo disperso a $9 \mathrm{~g} \mathrm{SSV/L}$ en comparación con las otras dos concentraciones, pero por debajo a los valores obtenidos con la FS. Los resultados con el lodo granular fueron similares entre las tres concentraciones de inóculo probadas (Figura 1); es decir la

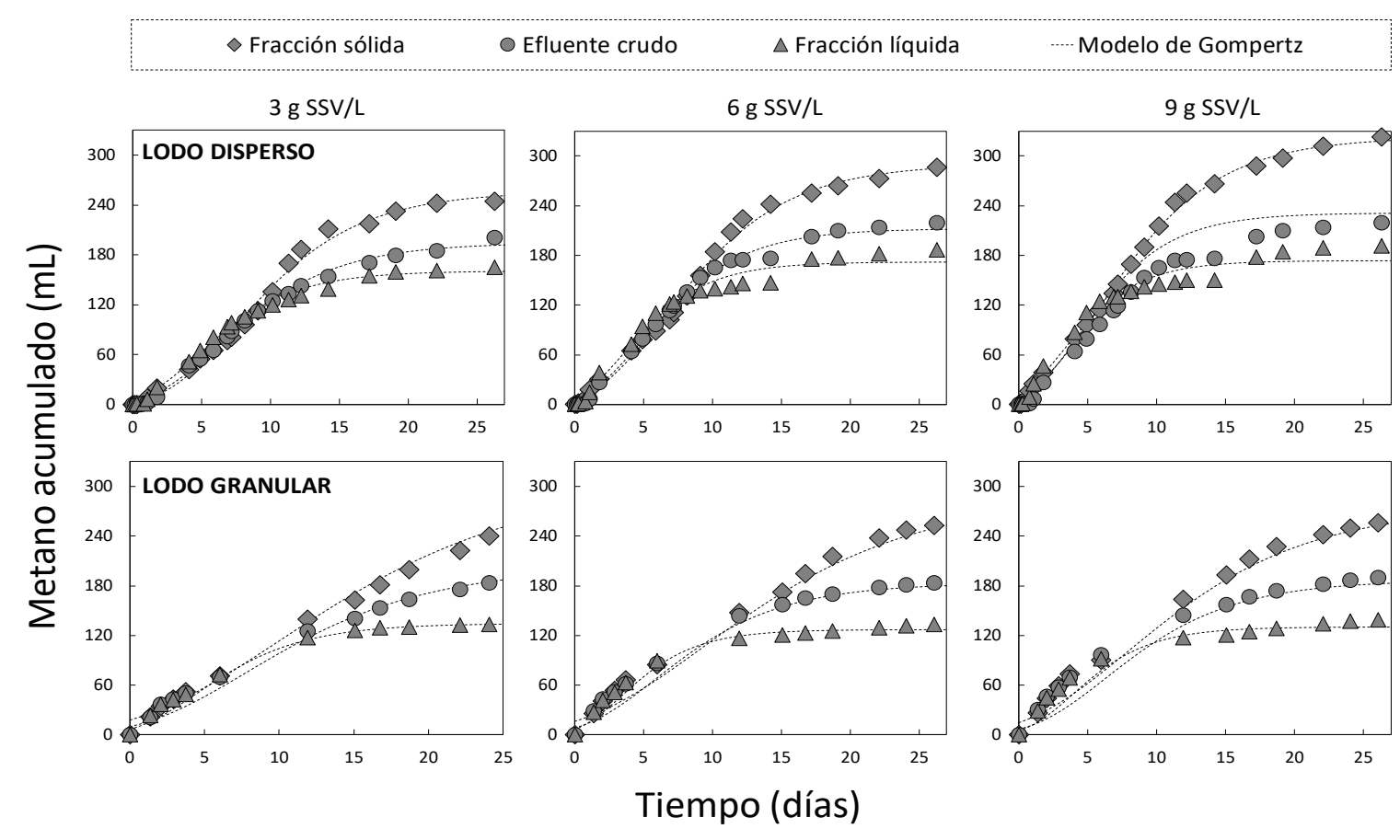

Figura 1. Producción de metano acumulado durante la digestión anaerobia de los tres tipos de agua residual porcina en presencia de diferentes concentraciones de lodo granular y lodo disperso.

Figure 1. Cumulative methane production during the anaerobic digestion of the three types of swine wastewater in the presence of different concentrations of granular and disperse sludge. 
cantidad de metano acumulado tuvo una diferencia entre los tratamientos no mayor a $\sim 7 \mathrm{~mL}$ para las concentraciones de lodo granular según cada fracción. La producción de metano con la FS a $9 \mathrm{~g} \mathrm{SSV/L} \mathrm{fue} 1.26$ veces mayor con el lodo granular $(318.8 \mathrm{~mL}$ ) en comparación a lo obtenido con el lodo granular $(253.6 \mathrm{~mL})$. En general, este comportamiento se mantuvo a 3 y $6 \mathrm{~g}$ SSV/L de lodo metanogénico, pero en proporción menor a lo observado con la concentración de 9 g SSV/L.

El consumo de la materia orgánica contenida en las diferentes fracciones se muestra en la Figura 2. En general, la eficiencia de consumo, expresada como DQO, fue mayor utilizando el lodo disperso en relación con el lodo granular. Sin embargo, el análisis estadístico indica que no existen diferencias significativas entre algunas condiciones probadas. Por ejemplo, para los cultivos con el EC, la eficiencia alcanzó $\sim 75 \%$ con ambos lodos, y no se presentaron diferencias significativas, con excepción de la concentración a $3 \mathrm{~g} \mathrm{SSV/L} \mathrm{con}$ el lodo disperso, que alcanzó $85 \%$ y que es estadísticamente diferente al resto. Los cultivos con la FS fueron en los que se presentaron las eficiencias de consumo de DQO más bajas, con valores que van desde $47.5 \%$ a $63.8 \%$. En este tipo de agua residual el consumo de DQO con el lodo granular no mostró diferencia estadística entre las tres concentraciones de inóculo, pero si fueron estadísticamente diferentes con los resultados obtenidos con el lodo disperso inoculados con 3 y $6 \mathrm{~g} \mathrm{SSV/L}$, alcanzando valores de consumo más altos (Figura 2). En los cultivos con la FL las eficiencias de consumo de DQO tuvieron un rango entre $71 \%$ a $89 \%$, alcanzándose los valores más altos para la concentración de $3 \mathrm{~g}$ SSV/L, tanto para el cultivo con el lodo granular y disperso, en donde no hay diferencia estadística significativa entre sus medias.

La separación sólida-líquida en el efluente porcino y su posterior tratamiento por digestión anaerobia incrementó la producción de metano (Figura 1). En estudios previos se ha observado que el rendimiento de metano se incrementó hasta 1.18 veces con la FS en relación a otras fracciones, utilizando aguas residuales de granjas de gestación y engorda (Cestonaro do Amaral et al., 2016), este incremento está por debajo del alcanzado en este estudio, que fue de hasta 1.47 veces mayor. Este resultado es esperado, ya que la muestra de agua residual con la FS contiene una mayor cantidad de materia orgánica en comparación a la FL (Tabla 1), lo cual es típico en los efluentes provenientes de granjas porcinas (Kebede-Westhead et al., 2006; Vanotti et al., 2018). Sin embargo, en otras muestras de agua residual porcina se ha encontrado que la $\mathrm{FL}$, en la cual previamente se eliminó el material floculante, fue la más apropiada para producir metano en términos de rendimiento (Rico et al., 2012; Yang et al., 2015). Esta diferencia en los resultados puede ser atribuida a la forma en la cual se utilizó la FS en el presente estudio, en donde la re-suspensión de los sólidos en el agua ocasionó que una fracción de la materia orgánica, que no necesariamente formaba parte de material particulado, contribuyó a tener una cantidad de DQO en forma soluble. Por otra parte, la presencia de antibióticos de uso veterinario en el agua residual porcina también puede ser responsable de inhibir la producción de metano, tanto en muestras con o sin sólidos.
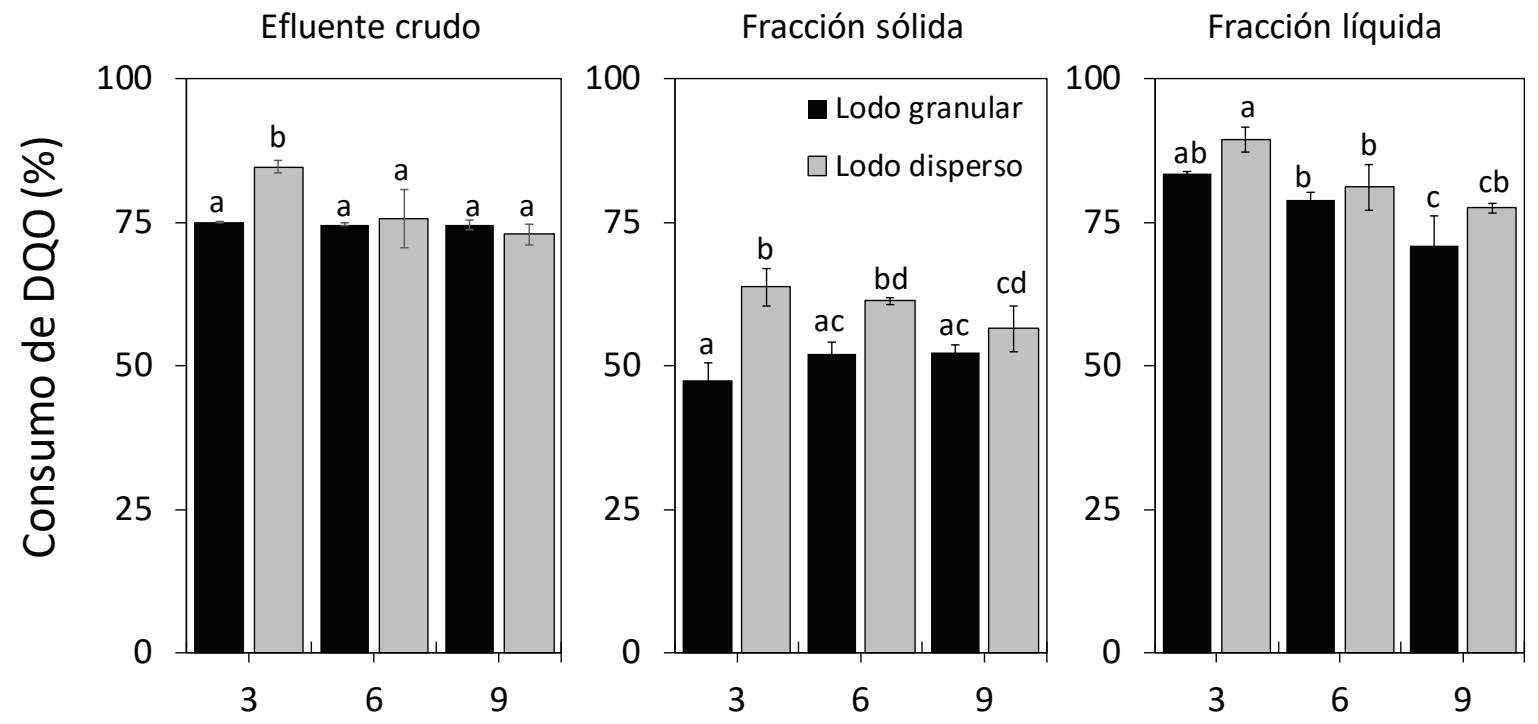

\section{Concentración de inóculo (g SSV/L)}

Figura 2. Eficiencia de consume de DQO en los cultivos con las diferentes muestras de agua residual porcina y concentraciones de inóculo. Letras diferentes arriba de cada barra indican diferencia significativa en las medias $(P<0.05)$, según el tipo de agua residual utilizada.

Figure 2. COD consumption efficiency in the cultures with the different samples of swine wastewater and inoculum concentrations. Different letters on each bar indicates a significant difference in means $(P<0.05)$, according to the type of wastewater used. 
Esto se debe a que la naturaleza química de ciertos antibióticos les permite tener mayor capacidad de solubilizarse en el agua, pero a otros una mayor afinidad de ser adsorbidos en el material particulado (Van Epps y Blaney, 2016); lo que puede influir en la cantidad de metano que se produce a partir de las fracciones sólidas y líquidas.

\section{Parámetros cinéticos a partir de modelo de Gompertz}

La estrategia de separación del efluente porcino en fracciones sólida y liquida, así como las distintas condiciones del inóculo tuvieron un impacto sobre la fase lag, $M_{\max }$ y $R_{\max }$ obtenidas con el modelo de Gompertz (Tabla 2). En general, para los tres tipos de agua residual, los valores de $M_{\max }$ con el lodo disperso fueron más altos que con el lodo granular, sin embargo, en algunos casos no hay diferencia estadística significativa entre los tipos de lodo usado. También, el resultado de $M_{\max }$ fue mayor utilizando la FS, aun cuando esta fracción tiene una concentración de DQO menor a la del EC. El valor de $M_{\max }$ con el EC y utilizando lodo disperso con $9 \mathrm{~g} \mathrm{SSV/L} \mathrm{fue}$ el más alto, alcanzando $231.6 \mathrm{~mL}$, pero es estadísticamente similar al obtenido con $6 \mathrm{~g} \mathrm{SSV/L}$ que alcanzó $212.3 \mathrm{~mL}$. En tanto, con el lodo disperso a $3 \mathrm{~g} \mathrm{SSV/L}$ no hubo diferencia significativa al compararlo con las tres concentraciones de lodo granular. Con la FS, el valor de Mmax más alto también

Tabla 2. Parámetros cinéticos obtenidos con el modelo de Gompertz tras la digestión anaerobia de los distintos tipos de agua residual, utilizando tres concentraciones de lodo disperso (LD) y lodo granular (LG).

Table 2. Kinetic parameters obtained from the Gompertz model after the anaerobic digestion of the different types of wastewaters, using three concentrations of disperse (LD) and granular sludge (LG).

\begin{tabular}{|c|c|c|c|c|c|}
\hline $\begin{array}{c}\text { Tipo de lodo y } \\
\text { concentración (g SSV/L) }\end{array}$ & Fase lag, (días) & $\begin{array}{l}M_{\max } \\
(\mathrm{mL})\end{array}$ & $\underset{\text { (mL/día) }}{\mathbf{R}_{\text {max }}}$ & $\mathbf{R}^{2}$ & Decremento en $M_{\max }$ \\
\hline \multicolumn{6}{|c|}{ Efluente crudo } \\
\hline $\operatorname{LD}(3)$ & $1.55+0.16 a$ & $191.7+11.2 \mathrm{ab}$ & $15.1+3.9 \mathrm{ab}$ & 0.9953 & -2.14 \\
\hline $\operatorname{LD}(6)$ & $0.92+0.19 b$ & $212.7+6.7$ ac & $19.5+1.1 \mathrm{ac}$ & 0.9958 & -2.17 \\
\hline $\operatorname{LD}(9)$ & $0.81+0.03 b$ & $231.6+17.4 c$ & $23.6+1.7 c$ & 0.9962 & -2.14 \\
\hline LG (3) & $0.76+0.14 b$ & $189.9+12.8 \mathrm{ab}$ & $10.7+2.8 b$ & 0.9956 & -2.18 \\
\hline LG (6) & $0.69+0.14 b$ & $180.0+2.0 \mathrm{~b}$ & $13.0+0.4 b$ & 0.9961 & -2.28 \\
\hline LG (9) & $1.05+0.02 b$ & $182.9+4.0 \quad b$ & $13.0+0.4 b$ & 0.9935 & -2.17 \\
\hline \multicolumn{6}{|c|}{ Fracción sólida } \\
\hline $\operatorname{LD}(3)$ & $2.43+0.75 a$ & $250.3+10.4 a$ & $18.8+1.6 a$ & 0.9957 & -- \\
\hline $\operatorname{LD}(6)$ & $1.49+0.33 a b$ & $285.7+13.4 b$ & $21.4+2.9 a b$ & 0.9961 & -- \\
\hline $\operatorname{LD}(9)$ & $1.06+0.16 b c$ & $318.8+15.5 c$ & $24.5+1.2 b$ & 0.9977 & -- \\
\hline LG (3) & $0.38+0.13 c$ & $258.1+10.4 a b$ & $11.8+1.8 c$ & 0.9962 & -- \\
\hline LG (6) & $0.53+0.15 b c$ & $251.2+7.3 a$ & $12.1+0.4 c$ & 0.9953 & -- \\
\hline LG (9) & $0.50+0.09 c$ & $253.6+7.7 a$ & $13.6+0.1 c$ & 0.9957 & -- \\
\hline \multicolumn{6}{|c|}{ Fracción líquida } \\
\hline $\operatorname{LD}(3)$ & $0.81+0.43 a$ & $160.8+13.1 a$ & $15.1+0.9 a$ & 0.9949 & -- \\
\hline $\operatorname{LD}(6)$ & $0.30+0.11 \mathrm{ab}$ & $171.7+7.3 a$ & $19.0+1.4 b$ & 0.9889 & -- \\
\hline $\operatorname{LD}(9)$ & $0.03+0.02 b$ & $173.5+2.4 a$ & $20.5+0.7 b$ & 0.9848 & -- \\
\hline LG (3) & $0.35+0.04 a b$ & $133.8+3.8 b$ & $12.2+1.2 c$ & 0.9966 & -- \\
\hline LG (6) & $0.29+0.12 a b$ & $127.2+2.4 b$ & $15.1+0.2 \mathrm{a}$ & 0.9942 & -- \\
\hline LG (9) & $0.61+0.11 a$ & $130.3+0.5 b$ & $14.8+0.9 \mathrm{ac}$ & 0.9899 & -- \\
\hline \multicolumn{6}{|c|}{$\begin{array}{l}\text { a Número de veces en que la producción de metano en el efluente crudo disminuye respecto a la suma del metano } \\
\text { producido por separado en la fracción sólida y fracción líquida, según tipo y concentración de lodo. } \\
\text { Los valores representan el promedio } \pm \text { desviación estándar. } \\
\text { Letras diferentes después de cada valor indican diferencia significativa en las medias }(P<0.05) \text {, según el tipo de agua } \\
\text { residual utilizada. }\end{array}$} \\
\hline
\end{tabular}


se obtuvo con el lodo disperso a una concentración de $9 \mathrm{~g}$ $\mathrm{SSV} / \mathrm{L}$, que estadísticamente es diferente a los valores obtenidos con los otros tratamientos de la FS. Para la FL, $\mathrm{M}_{\text {max }}$ es estadísticamente similar para las tres concentraciones de lodo disperso, pero mostraron diferencia respecto a las tres concentraciones del lodo granular (Tabla 2). La estrategia de separación y posterior digestión de la FS y FL permitió incrementar la cantidad total de metano acumulado en comparación a la digestión del EC. Es decir, en todos los casos la suma de $M_{\max }$ de las FS y FL es mayor a la cantidad de metano producido por el EC, si se comparan los resultados entra las mismas concentraciones, evidenciado por una producción entre 2.14 y 2.28 veces menor con el EC (Tabla 2).

De manera similar a la $M_{\max }$ la $R_{\max }$ presenta valores más altos tanto con el uso de lodo disperso y la FS (Tabla 2). Para ejemplo, los valores de $\mathrm{R}_{\text {max }}$ alcanzados con la FS inoculados con el lodo disperso se incrementaron 1.59, 1.76 y 1.80 veces en comparación con los cultivos con el lodo granular para las concentraciones de 3,6 y $9 \mathrm{~g} \mathrm{SSV/L}$, respectivamente, encontrando diferencia significativa entre ambos tipos de lodo. Los cultivos con el lodo disperso con 6 y $9 \mathrm{~g} \mathrm{SSV/L,} \mathrm{bajo}$ los tres tipos de agua residual, alcanzaron los valores de $\mathrm{R}_{\max }$ más altos y mostraron diferencia significativa con el resto de los tratamientos. La tasa especifica de producción de metano resultó mayor al utilizar el lodo disperso en comparación con el lodo granular para los tres tipos de muestras de agua residual. Además, se encontró que entre menor fue la concentración de inoculo utilizada, las tasas especificas fueron más altas (Figura 3). Por ejemplo, para la FS y lodo disperso a $3 \mathrm{~g} \mathrm{SSV/L}$, la tasa específica alcanzó $6.25 \mathrm{~mL} \mathrm{CH}_{4} / \mathrm{g} \mathrm{SSV}$-día, en contraste, con $9 \mathrm{~g} \mathrm{SSV/L}$ fue tan sólo de $2.72 \mathrm{~mL} \mathrm{CH} / \mathrm{g}$
SSV-día, valores que presentan diferencia estadística significativa. Para el lodo granular sucedió algo proporcionalmente similar, en donde a la concentración de inóculo más baja se alcanzaron $3.94 \mathrm{CH}_{4} / \mathrm{g} \mathrm{SSV}$.día, en tanto para $9 \mathrm{~g} \mathrm{SSV/L} \mathrm{fue}$ $1.51 \mathrm{CH}_{4} / \mathrm{g} \mathrm{SSV}$.día, que son estadísticamente diferentes. Al comparar las tasas especificas entre ambos lodos para los cultivos con la FS, hay una diferencia importante (hasta 80 $\%)$, y en todos los casos para este tipo de agua residual, la diferencia estadística es significativa. Sin embargo, para la FL la diferencia fue menor (hasta $38 \%$ ), encontrándose que no hay diferencia significativa, excepto para la FL inoculado con $3 \mathrm{~g} \mathrm{SSV} / \mathrm{L}$.

\section{Tratamiento de los efluentes porcinos}

El tratamiento de los efluentes generados en granjas porcinas no debe considerarse de manera trivial. En parte, la complejidad de este tipo de efluentes radica en su alto contenido de materia orgánica, en donde una fracción elevada se encuentra como material particulado, con presencia de componentes que pueden resultar tóxicos como el amonio y antibióticos. A pesar de esta condiciones limitantes, hay disponibles una cantidad importante de biotecnologías que pueden ser utilizadas para obtener energía y diferentes productos químicos de alto valor a partir de los residuos de granjas porcinas (Alvarez et al., 2019). Resulta relevante el hecho de que un número importante de granjas porcinas en México carece de sistemas de tratamiento de sus residuos líquidos y sólidos o bien operan de forma ineficiente, teniendo principalmente sistemas a base de lagunas de oxidación, pero que no cumplen los estándares mínimos de calidad y eficiencia (Cervantes et al., 2007; Garzón-Zúñiga y Buelna, 2014). De
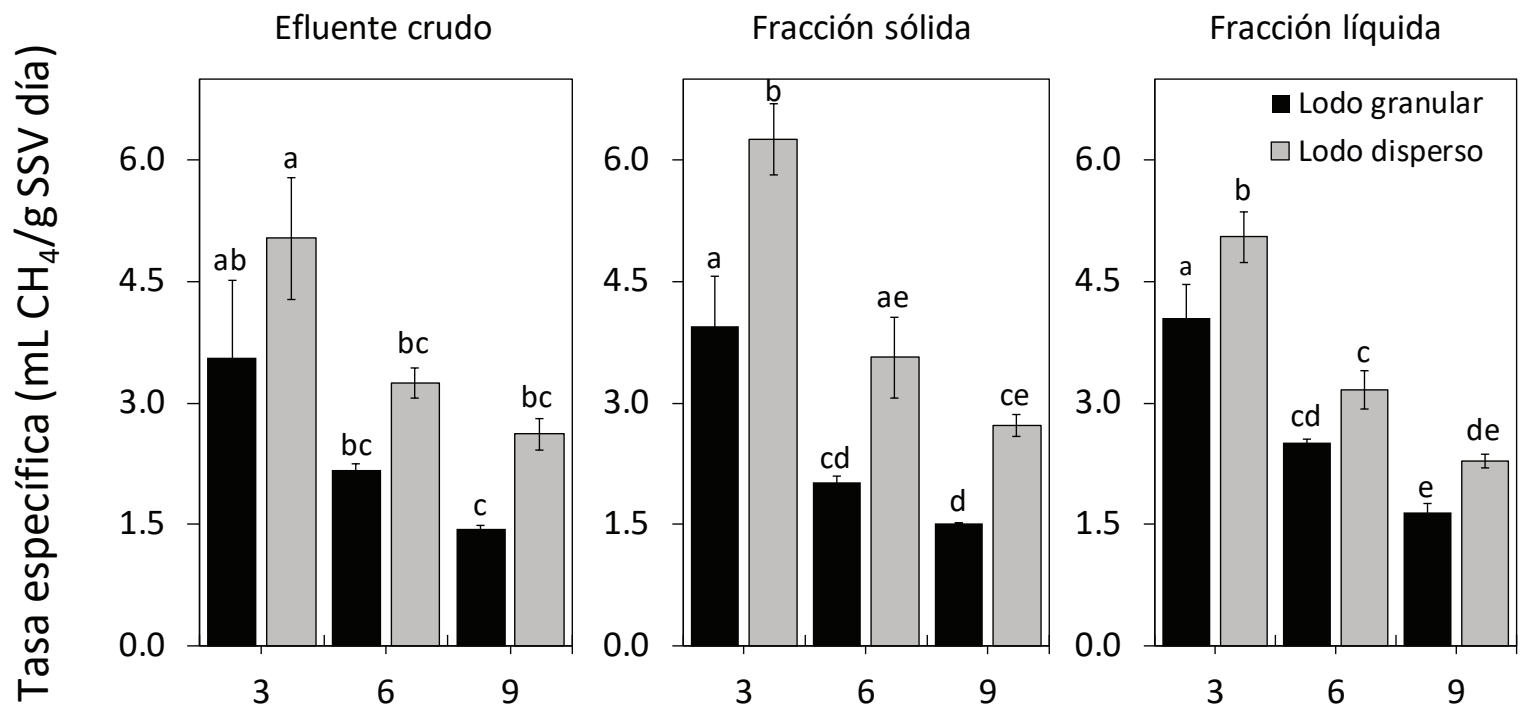

\section{Concentración de inóculo (g SSV/L)}

Figura 3. Tasa especifica de producción de metano a partir de los cultivos con las diferentes muestras de agua residual porcina y concentraciones de inóculo. Letras diferentes arriba de cada barra indican diferencia significativa en las medias $(P<0.05)$, según el tipo de agua residual utilizada.

Figure 3. Specific methane production rate from the cultures with the different samples of swine wastewater and inoculum concentrations. Different letters on each bar indicates significant difference in means $(P<0.05)$, according to the type of wastewater used. 
acuerdo a los resultados plasmados en este y otros estudios similares, la estrategia de separación de las fracciones sólidas y líquidas de los efluentes porcinos puede permitir mejorar la productividad de metano, la eficiencia de degradación de la materia orgánica, o bien la obtención de productos de alto valor como los mencionados por Alvarez et al. (2019).

En este estudio se utilizó lodo granular y lodo disperso, sin embargo, el principal propósito no fue identificar cual éstos dos tipos de inóculos presenta una mayor capacidad de degradación. En tanto, el objetivo fue evaluar el impacto de separar las diferentes fracciones del agua residual porcina durante la digestión anaerobia utilizando diferentes inóculos. El lodo granular y lodo disperso representan el tipo de inoculo utilizado en los reactores UASB y CSTR, respectivamente, que son dos de los sistemas de digestión anaerobia más representativos. La Figura 4 presenta dos propuestas de tratamiento de los residuos generados en granjas porcinas. En uno de los casos se plantea el uso de un sedimentador primario para la separación inicial de las fracciones del agua residual y su posterior tratamiento mediante procesos anaerobios en un reactor tipo UASB para la fracción líquida y un reactor CSTR para la fracción sólida (Figura 4A). En la Figura $4 \mathrm{~B}$ se plantea un tratamiento sin la separación de las fracciones, iniciando con la digestión anaerobia del efluente crudo en un reactor CSTR y posteriormente en un reactor UASB. En ambos casos de la Figura 4, el efluente del reactor UASB, conocido como digestato, puede utilizarse para riego agrícola debido a su contenido de nitrógeno y fósforo (Baştabak y Koçar, 2020). Además, el biogás generado en los reactores CSTR y UASB a partir de la digestión de las fracciones, puede ser utilizado para la generación de calor o electricidad (Kaparaju y Rintala, 2013), para el consumo en la granja o bien para proveer a pequeñas comunidades en función de la cantidad generada. Por último, los sólidos que se generen al final del proceso de tratamiento pueden ser utilizados para la producción de composta (Wang et al., 2018). Las propuestas de la Figura 4 son dos ejemplos de configuración de sistemas de tratamiento de los efluentes porcinos, pueden tenerse diferentes posibilidades dadas la necesidades, objetivos y recursos disponibles.

Los procesos centrales indicados en la Figura 4 son el uso de los reactores CSTR y UASB, en donde se pueden llevar a cabo todas las etapas de la digestión anaerobia, pero cuando se utilizan en un sistema de dos etapas consecutivas (CSTR-UASB), normalmente su función se especializa para los procesos de hidrólisis/acidogénesis y de metanogénesis, respectivamente (Van et al., 2020). El uso de los reactores para estos procesos de digestión anaerobia está en función

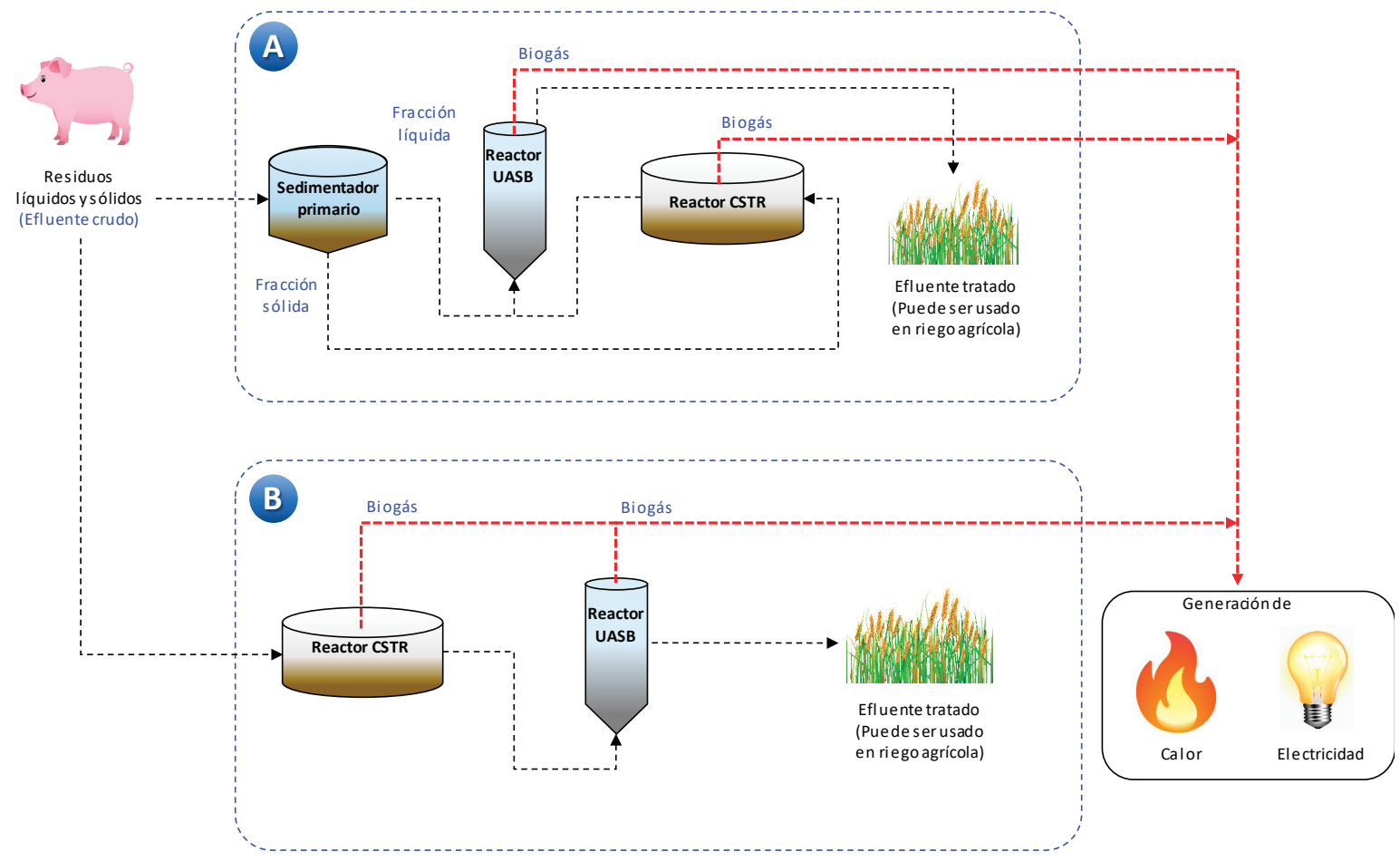

Figura 4. Estrategias biotecnológicas para tratar residuos sólidos y líquidos generados en granjas porcinas. Caso A: Pre-tratamiento inicial con un sedimentador primario para separar las fracciones sólidas y líquidas presentes en el efluente crudo. La fracción líquida es tratada en un reactor UASB y la fracción sólida en un reactor CSTR. El efluente del reactor CSTR se conduce al reactor UASB. Caso B: Tratamiento inicial del efluente crudo proveniente de la granja en un reactor CSTR, seguido de un reactor UASB que recibe la fracción liquida generada en el reactor CSTR. En ambos casos el efluente final puede ser aplicado en riego agrícola.

Figure 4. Biotechnological strategies to treat solid and liquid wastes produced in swine farms. Case A: Initial pre-treatment with a primary sedimentation tank to separate the solid and liquid fraction of the raw effluent. The liquid fraction is treated in a UASB reactor and the solid fraction in a CSTR reactor. The CSTR reactor effluent is conducted to the UASB reactor. Case B: Initial treatment of the raw effluent from the farm in a CSTR reactor, followed of an UASB reactor that receive the effluent from the CSTR reactor. In both cases, the final effluent can be used for agriculture irrigation. 
a las fracciones sólida/líquida a tratar, en tanto que en los reactores CSTR el sustrato puede contener más de $10 \%$ de sólidos, donde es requerida la etapa hidrolítica (Van et al., 2020), tal como los efluentes porcinos; los reactores UASB operan con sustratos con sólidos a una concentración $\leq 3 \%$, para favorecer la metanogénesis (Abbasi et al., 2011). Las propuestas de la Figura 4 consideran la digestión anaerobia inicial en dos etapas: un reactor CSTR (hidrólisis) seguido de un reactor UASB (metanogénesis), que permite optimizar las etapas de la digestión anaerobia a través del crecimiento de microorganismos especializados en sus ambientes óptimos y es más robusto que un sistema de una etapa al permitir fluctuaciones en la concentración de residuos (Van et al., 2020).

Aun cuando el reactor CSTR permite la digestión de la FS, una de sus desventajas es la necesidad de volúmenes grandes de reactor para alcanzar una cantidad de biomasa suficiente o bien largos tiempos de residencia hidráulica para degradar la materia orgánica (Van Lier et al., 2015). Además, el lodo disperso utilizado en los reactores CSTR puede lavarse con facilidad según las condiciones de mezclado, en tanto el lodo granular permanece intacto en el reactor UASB (Hulshoff Pol, 1989), que los ubica como reactores de alta densidad celular. Las ventajas de tener bacterias agregadas en forma de gránulos son: 1) permite que las poblaciones heterogéneas de microrganismos sintróficos se mantengan en condiciones fisiológicas favorables; 2) promueve fenómenos simbióticos; 3) incrementa la capacidad del consumo de nutrientes; 4) protege a las bacterias de organismos predadores; 5) disminución de la distancia de difusión para los intermediarios de la fermentación; y 6) protección de las células cuando existen condiciones poco favorables para el crecimiento (i.e. pH extremo) (Fitzpatrick et al., 1989). También, la construcción de reactores UASB requieren de un espacio menor de terreno, toleran altas cargas orgánicas y requieren menos tiempo de tratamiento (van Lier et al., 2015). Las ventajas que ofrecen los reactores CSTR y UASB permiten complementarse apropiadamente y los hacen sistemas de tratamiento apropiados para tratar efluentes porcinos.

\section{CONCLUSIONES}

La separación de sólida-líquida del agua residual porcina representa una estrategia para incrementar la eliminación de materia orgánica del agua. En este estudio, la separación de sólidos contribuyó en la eliminación de 69 \% de la DQO inicial. En el proceso de digestión anaerobia, la eficiencia de eliminación de DQO estuvo entre $47.5 \%$ y $89 \%$, en función de las condiciones experimentales probadas. La mayor cantidad de metano acumulado se produjo utilizando la FS, evidenciado por el incremento en $\mathrm{M}_{\max }$ entre 1.31 y 1.39 veces utilizando el lodo disperso y lodo granular, respectivamente, con relación a lo producido por el EC. Además, la cantidad total de metano que se produjo en conjunto por las FS y FL es mayor a la cantidad metano acumulado en el EC, lo cual evidencia que la separación sólida-líquida no sólo permite eliminar DQO del agua, sino que también mejora el proceso de digestión anaerobia. Los resultados de este estudio demuestran que es posible mejorar los sistemas de tratamiento de aguas residuales generadas en granjas porcinas aplicando tecnologías de fácil operación como los sedimentadores primarios. También, la aplicación de procesos anaerobios para sólidos, como los reactores CSTR, así como para los residuos líquidos en reactores tipo UASB, permitirá recuperar de manera más eficiente la energía a partir de la transformación de la materia orgánica en metano.

\section{AGRADECIMIENTOS}

Al Instituto Tecnológico de Sonora por el respaldo financiero otorgado para la realización de este estudio mediante el Programa de Fomento y Apoyo a Proyectos de Investigación (PROFAPI-2021).

\section{REFERENCIAS}

Abbasi, T., Tauseef, S., Abbasi, S. 2011. Biogas energy. In: Springer Briefs in Environmental Science. Springer Science and Business Media, New York.

Alvarez, L.H., García-Reyes, R.B., Ulloa-Mercado, R.G., Arellano Gil, M., García-González, A. 2019. Potencial biotecnológico para la valorización de residuos generados en granjas porcinas y cultivos de trigo. Entreciencias 7, 1-21.

APHA. 2005. Standard Methods for Examination of Water and Wastewater.

Barker, J.C., Zublena, J.P. 1996. Livestock manure nutrient assessment in North Carolina. North Carolina.

Baştabak, B., Koçar, G. 2020. A review of the biogas digestate in agricultural framework. J. Mater. Cycles Waste Manag. https://doi.org/10.1007/s10163-020-01056-9

Boursier, H., Béline, F., Paul, E. 2005. Piggery wastewater characterisation for biological nitrogen removal process design. Bioresour. Technol. 96, 351-358. https://doi. org/10.1016/J.BIORTECH.2004.03.007

Boxall, A.B.A., Kolpin, D.W., Halling-Sørensen, B., Tolls, J. 2003. Are veterinary medicines causing environmental risks? Environ. Sci. Technol. 37, 286A-294A.

Cervantes, F.J., Saldívar, J., Yescas, J.F. 2007. Estrategias para el aprovechamiento de desechos porcinos en la agricultura. Rev. Latinoam. Recur. Nat. 3, 3-12.

Cestonaro do Amaral, A., Kunz, A., Radis Steinmetz, R.L., Scussiato, L.A., Tápparo, D.C., Gaspareto, T.C. 2016. Influence of solid-liquid separation strategy on biogas yield from a stratified swine production system. J. Environ. Manage. 168, 229-235. https://doi.org/10.1016/j.jenvman.2015.12.014

Cioabla, A.E., Ionel, I., Dumitrel, G.A., Popescu, F. 2012. Comparative study on factors affecting anaerobic digestion of agricultural vegetal residues. Biotechnol. Biofuels 5 . https://doi.org/10.1186/1754-6834-5-39

Dourmad, J.Y., Ryschawy, J., Trousson, T., Bonneau, M., Gonzàlez, J., Houwers, H.W.J., Hviid, M., Zimmer, C., Nguyen, T.L.T., Morgensen, L. 2014. Evaluating environmental impacts of contrasting pig farming systems with life cycle assessment. Animal 8, 2027-2037. https://doi.org/10.1017/ S1751731114002134

FIRA, 2020. Panorama Agroalimentario: Carne de Cerdo.

Fitzpatrick, J.A., Roam, G.D., You, B.T. 1989. Anaerobic microbial aggregation and model development of a UASBR, in: Symposium on Anaerobic Digestion. Taipei, Taiwan, pp. 1518. 
Garzón-Zúñiga, M.A., Buelna, G. 2014. Caracterización de aguas residuales porcinas y su tratamiento por diferentes procesos en México. Rev. Int. Contam. Ambient. 30, 65-79.

Hanselman, T.A., Graetz, D.A., Wilkie, A.C. 2003. Manure-borne estrogens as potential environmental contaminants: a review. Environ. Sci. Technol. 37, 5471-5478.

Hulshoff Pol, L. 1989. The phenomenon of granulation of anaerobic sludge. Wageningen University, Wageningen.

Kaparaju, P., Rintala, J. 2013. Generation of heat and power from biogas for stationary applications: Boilers, gas engines and turbines, combined heat and power (CHP) plants and fuel cells, in: The Biogas Handbook: Science, Production and Applications. Elsevier Inc., pp. 404-427. https://doi. org/10.1533/9780857097415.3.404

Kebede-Westhead, E., Pizarro, C., Mulbry, W.W. 2006. Treatment of swine manure effluent using freshwater algae: Production, nutrient recovery, and elemental composition of algal biomass at four effluent loading rates. J. Appl. Phycol. 18, 41-46. https://doi.org/10.1007/s10811-005-9012-8

Rico, C., Rico, J.L., García, H., García, P.A. 2012. Solid - Liquid separation of dairy manure: Distribution of components and methane production. Biomass and Bioenergy 39, 370-377. https://doi.org/10.1016/j.biombioe.2012.01.031

Tomei, M.C., Braguglia, C.M., Cento, G., Mininni, G. 2009. Modeling of Anaerobic Digestion of Sludge. Crit. Rev. Environ. Sci. Technol. 39, 1003-1051. https://doi. org/10.1080/10643380801977818
Van, D.P., Fujiwara, T., Tho, B.L., Toan, P.P.S., Minh, G.H. 2020. A review of anaerobic digestion systems for biodegradable waste: Configurations, operating parameters, and current trends. Environ. Eng. Res. 25, 1-17. https://doi.org/10.4491/ EER.2018.334

Van Epps, A., Blaney, L. 2016. Antibiotic Residues in Animal Waste: Occurrence and Degradation in Conventional Agricultural Waste Management Practices. Curr. Pollut. Reports. https:// doi.org/10.1007/s40726-016-0037-1

Van Lier, J.B., van der Zee, F.P., Frijters, C.T.M.J., Ersahin, M.E. 2015. Celebrating 40 years anaerobic sludge bed reactors for industrial wastewater treatment. Rev. Environ. Sci. Biotechnol. https://doi.org/10.1007/s11157-015-9375-5

Vanotti, M.B., Ro, K.S., Szogi, A.A., Loughrin, J.H., Millner, P.D. 2018. High-Rate Solid-Liquid Separation Coupled With Nitrogen and Phosphorus Treatment of Swine Manure: Effect on Water Quality. Front. Sustain. Food Syst. 2, 49. https://doi. org/10.3389/fsufs.2018.00049

Wang, K., Mao, H., Wang, Z., Tian, Y. 2018. Succession of organics metabolic function of bacterial community in swine manure composting. J. Hazard. Mater. 360, 471-480. https://doi. org/10.1016/j.jhazmat.2018.08.032

Yang, D., Deng, L., Zheng, D., Liu, G., Yang, H., Wang, L. 2015. Separation of swine wastewater into solid fraction, concentrated slurry and dilute liquid and its influence on biogas production. Fuel 144, 237-243. https://doi. org/10.1016/J.FUEL.2014.12.044 